\title{
Platelet gel and fibrin sealant reduce allogeneic blood transfusions in total knee arthroplasty
}

\author{
P. A. M. Everts ${ }^{1}$, R. J. J. Devilee ${ }^{2}$, C. Brown Mahoner ${ }^{3}$, M. Eeftinck-Schattenkerk ${ }^{2}$, H. A. M. Box ${ }^{1}$, J. T. A. Knape ${ }^{4}$ and \\ A. VAN ZUNDERT ${ }^{5}$ \\ Departments of ${ }^{1}$ Extra Corporeal Blood Management and ${ }^{2}$ Orthopaedic Surgery-Traumatology, Catharina Hospital Eindhoven, Eindhoven, the \\ Netherlands, ${ }^{3}$ Business School and Statistics, Winona State University, Winona, MN, USA, ${ }^{4}$ Department of Anaesthesiology, University Medical \\ Centre Utrecht, Utrecht, the Netherlands and ${ }^{5}$ Department of Anaesthesiology, Catharina Hospital Eindhoven, Eindhoven, the Netherlands
}

\begin{abstract}
Background: Total knee arthroplasty (TKA) is often associated with a considerable amount of post-operative blood loss, necessitating the transfusion of allogeneic blood, which can add to the complications. Optimization of strategies to reduce the need for blood transfusion is desired. This study was designed to evaluate the efficacy of autologous platelet gel and fibrin sealant in unilateral TKA.

Methods: Consecutive patients were operated on and assigned to the study and control groups. Study group patients $(n=85)$ were operated on according to our standard TKA protocol, with the application of autologous platelet gel and fibrin sealant on the wound tissues at the end of surgery. Eighty patients were operated on according to the same protocol, but without the use of platelet gel and fibrin sealant, and served as the control group. All blood transfusions, occurrence of wound leakage, wound healing disturbances and incidences of post-operative infections were recorded.

Results: Patients in the treatment group had a significantly higher post-operative haemoglobin level (11.3 vs. $8.9 \mathrm{~g} / \mathrm{dl}$, respectively) and a decreased need for allogeneic blood products
\end{abstract}

( 0.17 vs. 0.52 units, respectively) than those in the control group $(P<0.001)$. The incidences of wound leakage and wound healing disturbance were significantly less $(P<0.001)$ in patients managed with platelet gel and fibrin sealant. Four patients in the control group, who received blood products, developed wound infection. The hospital stay was decreased by $1.4 \pm 1.5$ days for patients in the treatment group $(P<0.001)$.

Conclusion: Peri-operatively applied platelet gel and fibrin sealant may reduce the incidence of allogeneic blood transfusions and complications associated with TKA.

Accepted for publication 12 December 2005

Key words: blood transfusions; fibrin sealant; platelet gel; total knee arthroplasty.

(C) Acta Anaesthesiologica Scandinavica 50 (2006)
$\mathrm{T}$ OTAL knee arthroplasty (TKA) is often associated with a considerable amount of post-operative blood loss, requiring allogeneic blood product transfusions (ABPTs) $(1,2)$. However, the potential health risks after ABPT include the development of blood-borne diseases, leading to transfusion-related non-infectious complications, and ABPTs contribute to the incidence of immunosuppression, which may lead to increased post-operative bacterial infection rates (3-6). Finally, human error has been associated with the administration of blood products, leading to critical, even life-threatening, problems (7). Increased awareness of these complications by surgical teams has led to a continuous search for blood conservation techniques in orthopaedic surgery. Alternatives to avoid transfusions include pre-operative autologous blood donation, epoetin alpha (EPO), intravenous iron, non-steroidal anti-inflammatory drugs, peri-operative haemodilution, intra-operative and post-operative blood cell salvage and hypotensive anaesthesia (8-11). However, these alternatives concentrate primarily on decreasing post-operative blood loss and thus avoiding the transfusion trigger. A more logical blood management strategy would be to improve haemostasis directly during and after surgery to reduce post-operative blood loss and ABPT, and improve wound healing, and, as a result, decrease the incidence of post-operative infections.

Platelet gel (PG) is manufactured from plateletrich plasma (PRP), which is obtained after the sequestration of a unit of autologous whole blood with a blood cell separator. Treatment with PG involves direct application of concentrated platelets and their growth factors, specifically plateletderived growth factor (PDGF) and transforming 


\section{P. A. M. Everts et al.}

growth factor- $\beta$ (TGF- $\beta$ ), which have favourable effects on the augmentation of the wound healing cascade $(12,13)$. Fibrin sealants (FSs) have been used in a variety of surgical specialities, such as cardiothoracic surgery, cosmetic surgery and neurosurgery, to reduce bleeding $(14,15)$. In orthopaedic surgery, FSs have mainly been used as reconstructive supportive surgical tools $(16,17)$. Only a few publications have addressed the use of FS as a haemostatic tool during TKA, and investigators in these studies used a non-autologous cryoprecipitatebased fibrinogen $(18,19)$. Our concept of perioperative blood management has involved the exogenous application of PG and FS at the surgical wound site. In this study, we evaluated the haemostatic efficacy of sealing tissues, vessels and lymphatics by combining the use of both PG and FS in patients undergoing primary TKA.

\section{Materials and methods}

Using a prospective observational study, we evaluated 165 patients who were scheduled for primary unilateral TKA and who underwent the same surgical procedure. The study was reported to the hospital ethical committee and, prior to surgery, all study patients were informed about the blood management procedure. Initially, 80 patients, who served as controls, were operated on without the use of blood management techniques. Thereafter, 85 patients were operated on and managed with our blood management protocol consisting of PG and FS application (study group). All patients were operated on consecutively. They all had osteoarthritis of the knee and were operated on under 'standard' spinal anaesthesia by two surgeons using an identical surgical protocol.

\section{Blood management product preparation}

Intra-operatively, after spinal anaesthesia had been administered, approximately $350 \mathrm{ml}$ of whole blood was pre-donated from the median cubital vein into a blood bag containing citrate for anti-coagulation. The autologous blood was then centrifuged in a $125-\mathrm{ml}$ Latham bowl of the blood cell separator (Electa, Sorin Group, Mirandola, Italy) in the operating room. The blood cell separator sequestered the whole blood in a semi-automatic controlled operating mode via a hard spin at a $g$ force of 1660 . As a result, the whole blood in the centrifuge was fractionated into blood components, a process dependent on the weight of the individual blood components. Initially, the plateletpoor plasma (PPP) was evacuated from the whole blood and separated from the buffy coat layer and the erythrocytes. During this PPP release cycle, $20 \mathrm{ml}$ of the total PPP fraction was collected in a syringe for application at the wound site. The remaining PPP volume was collected in a blood bag and re-transfused during wound closure. During the second phase of the preparation process, the centrifuge was slowed to a $350 \mathrm{~g}$ soft spin cycle to collect the buffy coat layer, which consists of PRP and leucocytes (Fig. 1). PRP was entirely collected in a $60-\mathrm{ml}$ syringe, and was totally used for PG application. Finally, after the collection of PRP, the erythrocytes were collected in a transfusion bag and were reinfused to the patients during surgery at a time determined by the anaesthesiologist.

The addition of thrombin and calcium chloride to PPP results in the activation of the coagulation cascade by converting the fibrinogen in PPP to fibrin. This produces a stable fibrin cloth at the wound site, independent of the patient's coagulation process, resembling the final pathway of the physiological clothing cascade. PRP is also activated with thrombin and calcium, forming a viscous PG in which platelets subsequently degranulate and release their platelet growth factors.

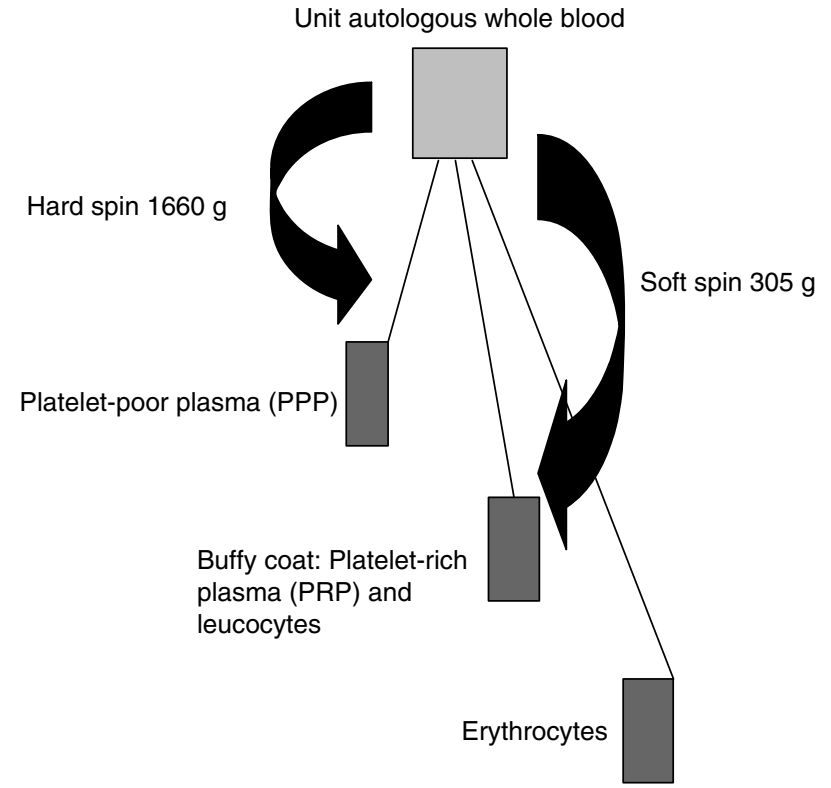

Fig. 1. Preparation of the blood components after autologous whole blood pre-donation in the blood cell separator. One unit of whole blood is separated first by a hard spin into platelet-poor plasma. Thereafter, the soft spin centrifugation process evacuates the buffy coat layer including platelet-rich plasma and leucocytes. Finally, erythrocyte blood cells are collected separately in a re-transfusion bag. 
In $85 \%$ of all patients treated with blood management, we were able to produce autologous thrombin (AT) to activate PRP and PPP. AT was produced by mixing $8 \mathrm{ml}$ of PRP with $0.35 \mathrm{ml}$ of calcium chloride $10 \%$ to antagonize the anti-coagulative effect of the citrate present in the pre-donation blood collection bag. This mixture was injected into a glass Petri dish which activates platelets to form a cloth. After 25-40 min, a cloth was formed inside the glass dish. This cloth was manually squeezed prior to PG and FS application. The cloth debris was left behind in the dish and the liquid part, containing AT (15-30 IU), was aspirated in a syringe. PRP and PPP were activated with the addition of AT at a ratio of $1 \mathrm{ml}$ AT to $4 \mathrm{ml}$ of blood components.

In 12 patients, we were unable to produce $\mathrm{AT}$, and therefore had to use bovine thrombin (ThrombinJMI, GenTrac Incorporated, Middleton, WI) at a concentration of $500 \mathrm{IU} / \mathrm{ml}$ to activate the blood components (20). During the period of the study (2002-2004), no restrictions with regard to the activation of PRP or PPP with bovine thrombin were effective in the Netherlands.

\section{Operative procedure}

Both cemented (Advantim and Advance, Wright Medical Products, Arlington, TN and Somas, the Netherlands) and non-cemented (LCS, Johnson and Johnson DePuy, Piscataway, NJ) prostheses were placed in a bloodless field with the use of a pneumatic tourniquet. Haemostasis, after the prosthesis had been inserted and the tourniquet deflated, was performed with the use of electrocautery of the major blood vessels. Before wound closure, the knee soft tissues and joint were rinsed with saline solution to remove all debris. In the study group, the entire operative field was thoroughly dried with gauze before $10 \mathrm{ml}$ of PG was initially injected in the back of the knee cavity, the posterior recess, the gutters and the raw exposed surfaces of the femur and tibia. The wound area was retracted and FS was applied to the dried tissues by a double syringe technique and delivered by aerosol topical spraying (Aerosol Ratio Applicator, Micromedics Inc, St. Paul MN) at a distance of $15 \mathrm{~cm}$. The wound was closed in layers. After closure of the knee capsula, another $10 \mathrm{ml}$ of PG was injected between the stitches on the repaired extensor mechanism and the prepatellar fat. The closed capsula and the subcutaneous tissues were then sprayed again with FS. No wound drains were used in the joints of patients in whom blood management techniques were applied to prevent evacuation of the PG, and thus the platelet growth factors, via the wound drain. Control patients received a wound drain as a routine measure. After skin closure, a wound dressing and a compressing bandage were applied.

The following data were obtained in both groups: haemoglobin values were determined pre-operatively, on the first, second, third and fourth postoperative days and at hospital discharge. In the blood management group, platelet and leucocyte counts were performed pre-operatively and after blood sequestration in PRP to assess the platelet yield after the sequestration procedure.

The indication to give allogeneic blood products in this study was a haemoglobin level of less than $8.8 \mathrm{~g} / \mathrm{dl}$ to avoid oxygen transport deficiency which might affect post-operative rehabilitation and recovery. The number of homologous blood products transfused, any wound complications, wound dehiscence and signs of infection were recorded.

All patients received thrombosis prophylaxis via a subcutaneous injection of $20 \mathrm{mg}$ nadroparin (Fraxiparine), a low-molecular-weight heparin, daily, before the operation until 6 weeks postoperatively.

The primary objective of the study was to achieve a decrease in ABPTs following TKA. Our second objective was to monitor patient morbidity parameters which affected the hospital length of stay (e.g. joint function recovery, wound leakage, infection and wound healing disturbances), as judged by the ward physician. Wound leakage was determined by moist wound dressings after the second post-operative day. Wound healing disturbances were defined as mild rubor with mild oozing, but no skin dehiscence. After hospital discharge, all patients went home.

\section{Statistical methods}

The study data were recorded on customized patient tracking forms and entered into a computerized database that allowed unbiased and reliable data management. Statistical analysis utilized SAS statistical software (SAS Institute, Cary, NC, 2003). Categorical data were expressed as percentages with 165 patients included, and measured data were expressed as the mean \pm standard deviation.

Univariate analysis was performed using Fisher's exact test on all categorical data and Student's $t$-test on all measured data. Differences between means 


\section{P. A. M. Everts et al.}

were analysed using Student's $t$-test and Satterthwaite's approximation, which does not assume equal variances. A two-sided $P$ value of 0.05 or less was considered to be statistically significant. To compare means from two independent samples with $n_{1}$ and $n_{2}$ observations, where $m$ in this case is equal to zero:

$$
t=\frac{\left(\bar{x}_{1}-\bar{x}_{2}\right)-m}{s \sqrt{\frac{1}{n_{1}}+\frac{1}{n_{2}}}}
$$

The formula for Satterthwaite's approximation (21) for the degrees of freedom for the approximate $t$ statistic is:

$$
\begin{aligned}
\text { d.f. }= & {\left[\left\{\left(w_{1}+w_{2}\right)^{2}\right\} /\left(\left\{\left(w_{1}^{2}\right) /\left(n_{1}-1\right)\right\}+\left\{\left(w_{2}^{2}\right) /\right.\right.\right.} \\
& \left.\left.\left.\left(n_{2}-1\right)\right\}\right)\right]
\end{aligned}
$$

and provides a more conservative test of significance than the standard $t$-test.

\section{Results}

The study group patients $(n=85)$ and the control group $(n=80)$ were statistically homogeneous and comparable, with the exception of the weight, which was higher in the control group (Table 1).

The mean autologous pre-donation volume was $335 \mathrm{ml}( \pm 28 \mathrm{ml})$. The sequestration process of the blood cell separator resulted in an increase in platelet and leucocyte counts by $453 \%$ and $177 \%$, respectively, when compared with baseline values $(P<0.001)$.

The mean number of post-operative blood product transfusions was $0.17 \pm 0.6$ in the blood management treatment group compared with $0.52 \pm 0.9$ in the control group $(P<0.001)$. With regard to transfusion requirements, seven patients $(8.3 \%)$ in the blood management group received transfusions (15 units of erythrocytes) compared

Table 1

\begin{tabular}{lll}
\hline \multicolumn{2}{l}{ Patient characteristics and type of knee prosthesis used. } \\
\hline Description & $\begin{array}{l}\text { Treatment } \\
\text { group }(n=85)\end{array}$ & $\begin{array}{l}\text { Control } \\
\text { group }(n=80)\end{array}$ \\
\hline Age (years) & $69.4 \pm 9.1$ & $67.4 \pm 9.9$ \\
Gender (female/male) & $58 / 27$ & $58 / 22$ \\
Mean weight $(\mathrm{kg})$ & $74.0 \pm 10.7$ & $80.5 \pm 13.3^{\star}$ \\
Pre-operative & $13.6 \pm 1.1$ & $13.7 \pm 1.1$ \\
haemoglobin (g/dl) & & 73 \\
Cemented (\%) & 74 & \\
\hline
\end{tabular}

The values are given as the mean \pm standard deviation.

${ }^{\star} P<0.05$, the mean weight was significantly higher in the control group. with 22 patients $(27.5 \%)$ in the non-treated group (42 units of red packed cells) $(P<0.001)$. The majority of patients $(68 \%)$ received two units, $9 \%$ received more than three units and $22 \%$ received one unit. All transfused patients met the criterion to receive a blood transfusion.

The haemoglobin course is shown in Fig. 2. The decrease in haemoglobin at post-operative days 1 and 2 , relative to the baseline value, was significantly less in the blood management group than in the control group $(P<0.001$ and $P<0.01$, respectively).

At hospital discharge, all patients had a similar haemoglobin value in the blood management group $(10.9 \pm 1.0 \mathrm{~g} / \mathrm{dl})$ and in the control group $(10.5 \pm 1.1 \mathrm{~g} / \mathrm{dl})$.

In the group receiving PG and FS, two patients experienced wound leakage through their bandages. However, no superficial wound healing disturbances were seen in this group. In the control group, 12 patients experienced wound leakage $(P<0.001)$, and nine patients developed mild wound healing disturbances, with a $P$ value for the difference of 0.001 .

Superficial wound infections were observed in four patients in the control group vs. zero patients in the blood management group $(P<0.05)$. These comorbidity factors were associated with the total length of hospital stay, which was significantly shorter in the blood management group $(6.9 \pm 1.4$ days $)$ than in the control group $(8.3 \pm 2.9$ days $)(P<0.001)$.

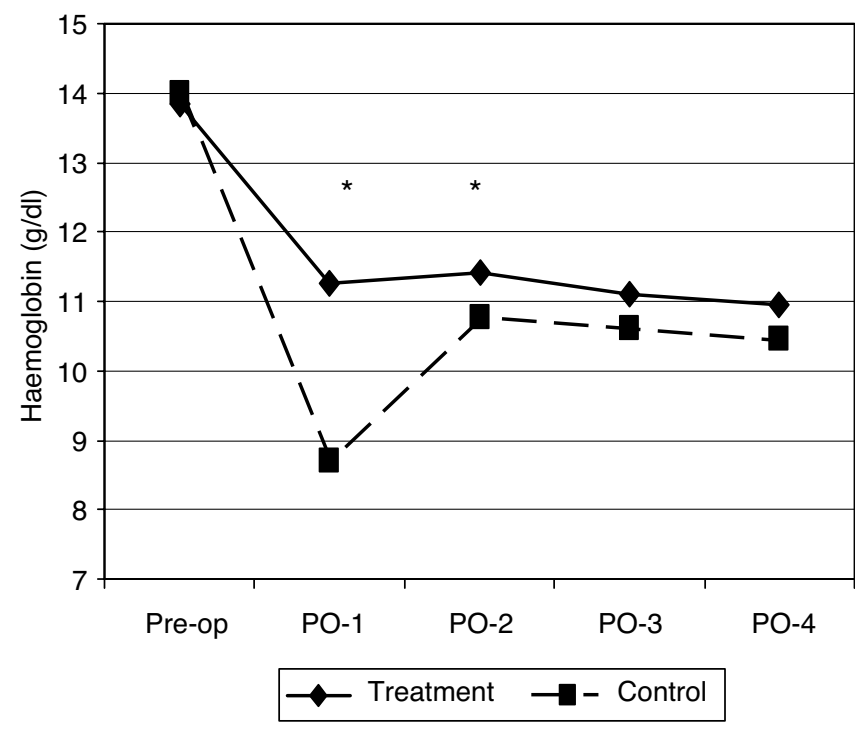

Fig. 2. Mean haemoglobin values for the treatment group and control group vs. the post-operative day (PO-1-4). ${ }^{*} P<0.001, a$ decrease in the haemoglobin loss for treated patients at PO-1 and PO-2. 


\section{Discussion}

TKA is associated with major blood loss. In this study, it was found that the application of PG and FS during TKA reduced significantly the need for allogeneic blood transfusions when compared with that required in control patients, using the same criteria for transfusion in both groups, although the average body weight, and thus the red blood cell mass, of the patients in the blood management group was significantly lower than that in the control patients. The decrease in haemoglobin and the need for blood transfusions in control patients were comparable with the data reported by others $(1,11)$. All transfusions were given on the first postoperative day.

The literature on FSs clearly shows improved haemostatic effects in both animal and human models (15); however, only a few similar clinical studies have been performed during TKA, and have shown results comparable to those obtained when haemostatic agents are used to control bleeding. Levy et al. (18) showed a decrease in blood loss and reduced blood transfusion requirements when using Quixil (Omrix Biopharmaceuticals SA, Brussels, Belgium), which he applied by topical spraying. In a multicentre, randomized controlled trial by Wang et al. (19), the same FS was used, and the decrease in haemoglobin level was almost 29\% less in the FS group than in the control group. In comparison with the above-mentioned studies, our blood management protocol involved the production of both autologous FS and PG. Activated PPP was sprayed as a tissue sealant on the edges of the sawn bones and soft tissues to avoid blood loss. Furthermore, FS may inhibit the inflammatory response, with less adhesion formation, as demonstrated by Jahoda et al. (22). It has been postulated that this decrease in inflammation may contribute to a lower tissue reaction, thus leading to fewer adhesions (23). Studies by Marx et al. (24) have confirmed the efficacy of concentrating platelets by centrifugation of whole blood and harvesting PRP. PG with a platelet concentration three to six times that of the native platelet concentration will release, amongst others, the platelet growth factors PDGF and TGF- $\beta$, which play a pivotal role in the wound healing process $(12,13)$. As PRP is a buffy coat product, it also contains a high concentration of leucocytes and, in particular, granulocytic neutrophils which contain myeloperoxidase (MPO), a substance known to remove bacteria (25). It may be speculated, therefore, that PG may also have anti-bactericidal properties.
Twelve patients required bovine thrombin because the autologously prepared thrombin failed to set up a firm gel. In the USA, bovine thrombin is still the PRP activator of choice to produce PG (26). However, at present, we have discarded the use of bovine thrombin in order to avoid the potential development of antibodies to clotting factors $\mathrm{V}$ and XI and thrombin, which might occasionally lead to life-threatening coagulopathies (27). The analysis of the study parameters from these patients did not reveal any differences in outcome relative to the patients treated with AT.

Blood loss in TKA occurs mainly after surgery, as the procedure is performed with the use of a pneumatic tourniquet. Because our primary objective was to reduce post-operative blood loss by means of peri-operative blood management techniques, the use of post-operative drainage and re-infusion of unwashed shed blood was obsolete. Furthermore, there is reasonable concern about the quality of unwashed re-transfused wound blood (28). It has also been argued that the use of FSs during joint replacement may be unnecessary because of autologous pre-donation techniques to compensate for post-operative blood loss. Although autologous pre-donated blood is not associated with the risks of viral disease transmission and immunomodulatory changes, the incidence of administrative transfusion errors and bacterial overgrowth is comparable with that when using homologous transfused blood $(7,29)$. Furthermore, a special blood banking protocol and schedule to pre-donate prior to surgery is required and certain patients are unable to donate blood. Increasing the pre-operative haemoglobin value through pre-operative EPO injections, in order to minimize the number of ABPTs by avoiding the transfusion trigger, has become more popular. However, the aim of our blood management concept is to avoid post-operative blood loss and to use the patient's own blood components to support the healing process. Furthermore, the costs of EPO outweigh those of our blood management programme. Our control patients received a wound drain. The orthopaedic surgeons did not use a postoperative drain in the study patients to prevent PG, and therefore platelet growth factors, being removed from the wound cavity. No differences were seen with regard to leg swelling and return of active function of the quadriceps muscle between treated and control patients. This finding is in accordance with the observations of several other authors, indicating that there is no difference in 
post-operative outcome when drained and nondrained patients are compared with regard to leg swelling, wound disturbances, return of function and wound leakage (30).

This study showed that there was a significant decrease in the incidence of wound leakage and wound healing disturbances in patients treated with PG and FS. The decreased leakage is most probably the result of the efficacy of FS in sealing the oozing soft tissue wound sites and of the ability of PG to plug the bone marrow sinusoids at the cut bone sites in the knee cavity and at surrounding sawn bone tissues in the femur and tibia next to the prosthesis. The reduced wound leakage presumably results in decreased wound healing disturbances, and wound healing is further supported by the growth factors released from PG.

Four patients in our control group received two units of allogeneic blood and developed a superficial wound infection which was treated with antibiotic therapy. None of the treated patients showed signs and symptoms of infection. Another explanation for the development of these wound infections could be the increased allogeneic transfusion rate in the control group. Our findings are in accordance with the data of Thomas et al. (11) and with the data from almost 4000 patients in the Orthopedic Surgery Transfusion Hemoglobin European Overview (OSTHEO) study, which showed that more than $4 \%$ of allogeneic transfused patients developed a wound infection (31). In a meta analysis, Hill et al. showed that allogeneic blood transfusions are associated with transfusion-associated immunomodulation, and that transfusions are an important risk factor for the development of postoperative bacterial infections (6). In addition, ABPT may lead to a decrease in pro-angiogenic factors (e.g. interleukin-8) that are essential for wound healing (32).

Although we attempted to reduce, as much as possible, confounding factors in our study (same anaesthetic technique, one type of blood cell separator and limited number of surgeons), the effect of varying platelet and fibrinogen concentrations was not studied. The study was not sufficiently powerful to demonstrate whether different concentrations of platelets and fibrinogen had an effect on haemoglobin loss and thus erythrocyte transfusions.

In conclusion, in patients undergoing TKA, we demonstrated that the exogenous application of PG and FS to the wound site supported the haemostatic condition with the delivery of concentrated platelet growth factors and leucocytes. This resulted in a decreased blood transfusion requirement, lower incidence of post-operative wound healing disturbances, less infections and a shorter length of hospital stay. Future studies should aim to elucidate the potential anti-bactericidal effect of PG, to demonstrate the economic costs and benefits and to address logistic problems in performing these blood management techniques, not only during TKA but potentially also in other orthopaedic surgeries.

\section{References}

1. Bierbaum BE, Callaghan JJ, Galante JO, Rubash HE, Tooms RE, Welch RB. An analysis of blood management in patients having a total hip or knee arthroplasty. J Bone Joint Surg Am 1999; 81: 2-10.

2. Berman AT, Geissele AE, Bosacco SJ. Blood loss with total knee arthroplasty. Clin Orthop 1988; 234: 137-8.

3. Goodnough LT, Brecher ME, Kanter MH, AuBuchon JP. Transfusion medicine. First of two parts-blood transfusion. N Engl J Med 1999; 340: 438-47.

4. Schreiber GB, Busch MP, Kleinman SH, Korelitz JJ. The risk of transfusion-transmitted viral infections. The Retrovirus Epidemiology Donor Study. N Engl J Med 1996; 334: 1685-90.

5. Blumberg N, Heal JM. Immunomodulation by blood transfusion: an evolving scientific and clinical challenge. Am J Med 1996; 101: 299-308.

6. Hill GE, Frawley WH, Griffith KE, Forestner JE, Minei JP. Allogeneic blood transfusion increases the risk of postoperative bacterial infection: a meta-analysis. I Trauma, Injury, Infect Crit Care 2003; 54 (7): 908-14.

7. Williamson LM, Lowe S, Love EM et al. Serious hazards of transfusion (SHOT) initiative: analysis of the first two annual reports. Br Med J 1999; 319: 16-9.

8. Canadian Orthopedic Perioperative Erythropoietin Study Group. Effectiveness of perioperative recombinant human erythropoietin in elective hip replacement. Lancet 1993; 341: 1227-32.

9. Clements DH, Thomas MD, Sulco P et al. Salvage and reinfusion of postoperative sanguineous wound drainage. J Bone Joint Surg 1992; 74: 646-51.

10. Nelson CL, Fontenot HJ. Ten strategies to reduce blood loss in orthopedic surgery. Am J Surg 1995; 170 (Suppl. 6A): 64S-68S.

11. Thomas D, Wareham K, Cohen D, Hutchings H. Autologous blood transfusion in total knee replacement surgery. $\mathrm{Br} J$ Anaesth 2001; 86 (5): 669-73.

12. Pierce GF, Mustoe TA, Lingelbach J et al. Platelet-derived growth factor and transforming growth factor $\beta$ enhance tissue repair activities by unique mechanisms. J Cell Biol 1989; 109 (1): 429-40.

13. Mustoe TA, Pierce GF, Thomason A, Gramates P, Sporn MB, Deuel TF. Accelerated healing of incisional wounds in rats induced by transforming growth factor- $\beta$. Science 1987; 237 (4820): 1333-6.

14. Kjaergaard HK, Fairbrother JE. Controlled clinical studies of fibrin sealant in cardiothoracic surgery - a review. Eur J Cardiothorac Surg 1996; 10: 727-33.

15. Mankad PS, Codispoti M. The role of fibrin sealants in hemostasis. Am J Surg 2001; 182: 21S-28S.

16. Angermann P, Riegels-Nielsen P. Fibrin fixation of osteochondral talar fracture. Acta Orthop Scand 1990; 61: 551-5.

17. Schlag G, Redl H. Fibrin sealant in orthopaedic surgery. Clin Orthop 1988; 227: 269-85. 
18. Levy O, Martinowitz U, Oran A, Tauber C, Horoszowski H. The use of fibrin tissue adhesive to reduce blood loss and the need for blood transfusion after total knee arthroplasty. A prospective, randomized, multicenter study. J Bone Joint Surg 1999; 81: 1580-8.

19. Wang GJ, Hungerford DS, Savory CG et al. Use of fibrin sealant to reduce bloody drainage and haemoglobin loss after total knee arthroplasty. J Bone Joint Surg 2001; 83 (10): 1503-5.

20. Patricia A, Nelson PA, Noël Powers J, Estridge TD. Serological analysis of patients treated with a new surgical hemostat containing bovine proteins and autologous plasma. J Biomed Mat Res 2001; 58 (6): 710-9.

21. Khuri AI, Mathew T, Nel DG. A test to determine closeness of multivariate Satterthwaite's approximation. J Multivariate Anal 1994; 51 (1): 201-9.

22. Jahoda AE, Albala DM, Dries DJ, Kovacs EJ. Fibrin sealant inhibits connective tissue deposition in a murine model of peritoneal adhesion formation. Surgery 1999; 125 (1): 53-9.

23. Toosie K, Gallego K, Stabile BE et al. Fibrin glue reduces intra abdominal adhesions to synthetic mesh in a rat ventral hernia model. Am Surg 2000; 66: 41-5.

24. Marx RE, Carlson ER, Eichstaedt RM, Schimelle SR, Strauss JE, Georgeff KR. Platelet-rich plasma: growth factor enhancement for bone grafts. Oral Surg Oral Med Oral Pathol Oral Radiol Endod 1998; 85 (6): 638-46.

25. Lincoln JA, Lefkowitz DL, Cain T et al. Exogenous myeloperoxidase enhances bacterial phagocytosis and intracellular killing by macrophages. Infect Immun 1995; 63 (8): 3042-7.

26. Marx RE. Platelet rich plasma: evidence to support its use. J Oral Maxillofac Surg 2004; 62: 489-96.
27. Zehnder JL, Leung LLK. Development of antibodies to thrombin and factor $\mathrm{V}$ with recurrent bleeding in a patient exposed to topical bovine thrombin. Blood 1990; 76: 2011-6.

28. Southern EP, Huo MH, Mehta JR, Keggi XJ. Unwashed wound drainage blood. What are we giving our patients? Clin Orthop Rel Res 1995; 320: 235-46.

29. Landers DF, Hill GE, Wong KC, Fox IJ. Blood transfusion-induced immunomodulation. Anesth Analg 1996; 82: 197-204.

30. Ritter MA, Keating EM, Faris PM. Closed wound drainage in total hip or total knee replacement. A prospective, randomized study. J Bone Joint Surg 1994; 76 (1): $35-8$.

31. Rosencher N, Kerkkamp HE, Macheras G et al. OSTHEO Investigation: Orthopedic Surgery Transfusion Hemoglobin European Overview (OSTHEO) study: blood management in elective knee and hip arthroplasty in Europe. Transfusion 2003; 43 (4): 459-69.

32. Okano T, Ohwada $\mathrm{Y}$, Sato $\mathrm{N}$ et al. Blood transfusions impair anastomotic wound healing, reduce luminal-dependent chemiluminescence and increase interleukin-8. HepatoGastroenterology 2001; 48 (42): 1669-74.

Address:

Peter A. M. Everts

Catharina Hospital

Department of Extra-Corporeal Blood Management

Michelangelolaan 2, 5623 EJ, Eindhoven

the Netherlands

e-mail: everts@elive.nl 